\begin{tabular}{|c|c|c|}
\hline Beitr. Ent. & Keltern & ISSN 0005-805X \\
\hline $\mathbf{6 0}(2010) 2$ & S. $247-257$ & 20.12 .2010 \\
\hline
\end{tabular}

\title{
Prof. Dr. Holger H. Dathe als Entomologe und Direktor des Senckenberg Deutschen Entomologischen Instituts anlässlich seines 65. Geburtstages
}

Mit 1 Figur

Bernhard Klausnitzer

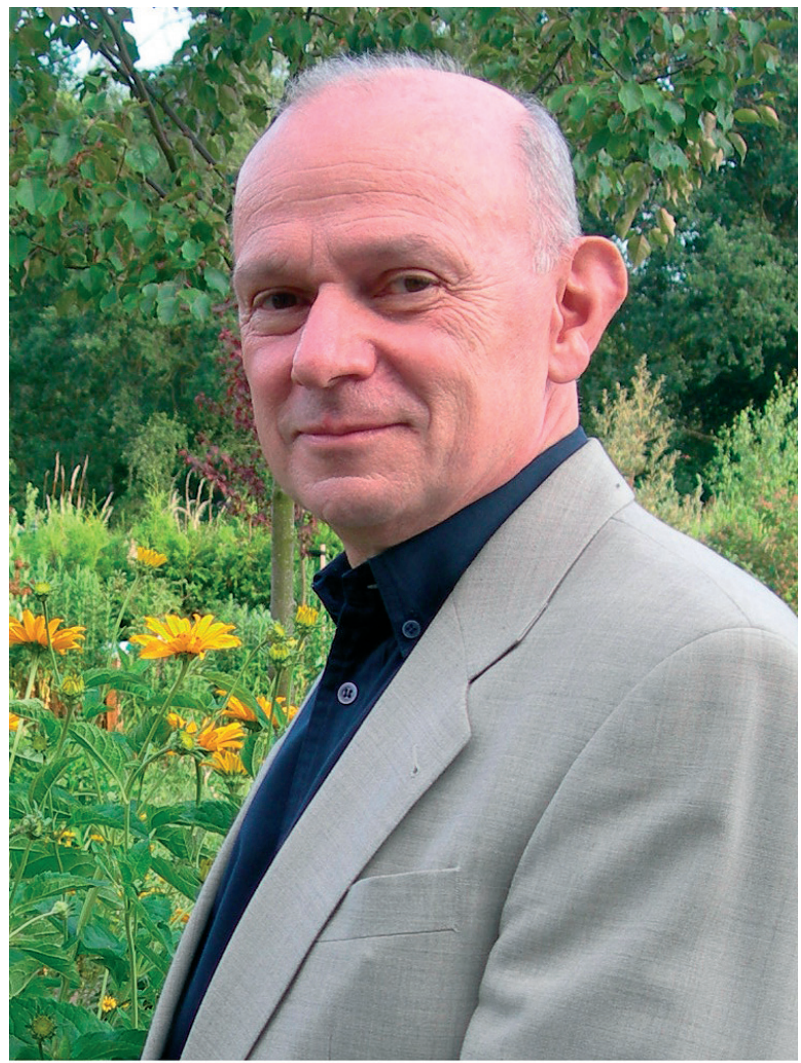

Foto: M. Dannowski 2010

Holger Dathe ist aus mehreren Gründen ein bekannter Entomologe. Zum Beleg soll an erster Stelle die Herausgabe der 2. Auflage der „Insecta“ im Lehrbuch der Speziellen Zoologie (2003, 2005: XIV + 961 Seiten, 564 Abbildungen) genannt werden. Das Buch hat sich als ein unentbehrliches Standardwerk erwiesen. Es ist geradezu ein Meilenstein in der entomologischen Literatur der Gegenwart, das sowohl bewährtes als auch in der Entwicklung befindliches Wissen in einem guten Verhältnis darstellt. Die Mühen des Herausgebers um eine ausgewogene, vergleichbare 
Gestaltung der einzelnen Kapitel bei höchster Kompetenz des Inhaltes - der Schwerpunkt sind aktuelle Sichten auf die phylogenetischen Beziehungen innerhalb der Taxa - sind immens gewesen. Es war gewiss nicht einfach, einen großen Kreis kompetenter Autoren zur Mitarbeit zu gewinnen und freundlich, aber nachdrücklich zu einer gewissen Einheitlichkeit ihrer Kapitel zu führen; das ist „Hohe Schule“, wenn man die starke Individualität der Entomologen bedenkt.

Der zweite Grund liegt in seinem Wirken als Hymenopterologe. Holger Dathe ist immer bemüht gewesen, die gesamte Formenfülle der Hymenoptera zu überschauen. Davon legt u. a. die Bearbeitung der riesigen und überaus vielfältigen Ordnung im eben genannten Lehrbuch Zeugnis ab. Auf 66 Seiten wird ein Überblick über das Weltwissen gegeben, angereichert durch eine fundierte eigene Sicht und zahlreiche originale Gedanken. Auch Band 4 (Hymenoptera) der „Entomofauna Germanica“ (herausgegeben von H. H. Dathe, A. Taeger \& S. M. Blank 2001) ist hier zu erwähnen. Es ist die erste vollständige Auflistung aller Hymenoptera Deutschlands! Über 90 Hymenopterologen haben zu diesem großartigen Gemeinschaftswerk ihr gesammeltes Wissen beigetragen.

Selbstverständlich musste innerhalb der Hymenoptera eine Spezialisierung erfolgen. Die Bienen (Anthophila) waren die Gruppe der Wahl. Eine größere Zahl von Publikationen, oft in Gemeinschaft mit anderen Hymenopterologen, behandeln dieses Taxon. Beispiele sind die Roten Listen für Brandenburg und Deutschland (mit C. Saure und P. Westrich), ein „Katalog der Bienen Österreichs, Deutschlands und der Schweiz“ (gemeinsam mit M. Schwarz, F. Gusenleitner \& P. Westrich, 1996). Im Jahre 1997 folgte ein mit Paul Westrich verfasstes und 1998 ergänztes Verzeichnis der Bienenarten Deutschlands. Zu nennen sind außerdem seine Bestimmungstabellen in der 9., 10. und 11. Auflage der „Exkursionsfauna für Deutschland“ Band 2. Herausragend ist die Bearbeitung der Bienen-Familien im Projekt „Arthropod Fauna of the UAE“, die er koordinierend, aber auch anleitend mit neun anderen führenden Spezialisten vorgelegt hat. Übrigens kam er gar nicht mehr umhin, seinen zweiten Vornamen als Autor mit zu nutzen, denn „H. Dathe“ war in der zoologischen Literatur durch seinen Vater „präokkupiert“.

Holger Dathe konzentrierte sich - um auch taxonomisch wirksam werden zu können - auf eine Teilgruppe, die Maskenbienen (Gattung Hylaeus). Seine gründlichen, mit großer Umsicht und Intensität ausgearbeiteten Publikationen schufen ihm im illustren Zirkel der führenden Forscher bald einen geachteten Platz, wobei der Linzer Hymenopterologen-Kreis eine zentrale fördernde Rolle spielte. Die erste Arbeit über Hylaeus mit einer Neubeschreibung erschien 1977, 24 zum Teil recht umfangreiche Veröffentlichungen folgten, z. B. über Arten aus China, Sibirien, arabischen Ländern, Kreta, der Iberischen Halbinsel und den Kanarischen Inseln. Präzise transpaläarktische Studien, nicht auf Europa beschränkt, ragen besonders heraus, z. B. über den Iran (1979), den Kaukasus (1980) und die Mongolei (1986). Bis dato beschrieb Holger Dathe 51 neue Arten aus der Gattung Hylaeus (siehe untenstehende Liste). Seine Bestimmungstabelle aus dem Jahre 1980 gehört noch immer zur Standardliteratur. Sie wurde ergänzt und erweitert durch einen Schlüssel für die auf der Iberischen Halbinsel vorkommenden Arten (2004). Charles Michener hat ihn als einen der (wenigen) Koautoren eingeladen, für sein großes Werk „The Bees of the World“ (2000, 2007) die paläarktischen Untergattungen von Hylaeus zu bearbeiten.

Der dritte Grund für seine hohe Präsenz liegt in seiner Verantwortung für das Deutsche Entomologische Institut über 17 Jahre hinweg.

Das Deutsche Entomologische Institut hatte in seiner 124-jährigen Geschichte immer wieder schwierige Perioden zu überstehen gehabt, oft stand die Existenz auf des Messers Schneide. Es hat nur jeweils relativ kurze Perioden gegeben, in denen die Mitarbeiterinnen und Mitarbeiter des Deutschen Entomologischen Instituts das Gefühl haben konnten, ihr Institut ist gesichert, ihre Arbeit rundum erwünscht. Viele Potenzen sind verschwendet worden in Begründungen der 
Existenzberechtigung des Hauses, der Sinnhaftigkeit der eigenen Arbeit. Dennoch ist erstaunlich, wieviel Bleibendes von Weltgeltung trotzdem - oder vielleicht auch gerade deshalb - geschaffen wurde.

Als Holger Dathe am 01.12.1993 die Leitung des Deutschen Entomologischen Instituts übernahm, zunächst kommissarisch, stand der elementare Erhalt der Einrichtung im Mittelpunkt. Bei der Auflösung der Akademie der Landwirtschaftswissenschaften im Zuge der deutschen Einheit war eine Kernmannschaft zusammengeblieben, weitere Mitarbeiter waren zeitweise über Projekte angestellt. Über eine mögliche dauerhafte Förderung sollte erst ein weiteres Begutachtungsverfahren entscheiden. Von Monat zu Monat wurde im Ministerium über die Finanzierung entschieden, eine längerfristige Planung war nicht möglich. Es ist nur schwer zu ermessen, kaum adäquat darzustellen, welches Maß an Kraft, diplomatischem Geschick und Optimismus nötig waren, um diese fast zehnjährige Periode zum guten Ende zu führen. Ganz entscheidend waren in dieser Zeit der Rückhalt in der Belegschaft und die wohlwollende, z. T. sehr engagierte Unterstützung von Personen außerhalb des Instituts, die von der Bedeutung und dem Wert des Deutschen Entomologischen Instituts zutiefst überzeugt waren.

Als Nachteil erwies sich die historisch begründete Anbindung des Instituts an die Agrarwissenschaft. Schon früh erwuchsen aus dieser Situation Zwänge, die nie befriedigend gelöst werden konnten. Seitens der Landwirtschaft gab es stets durchaus verständliche Forderungen nach der Unterstützung, gar selbstständigen Aufnahme von Forschungsarbeiten mit entsprechenden Zielstellungen, die im Deutschen Entomologischen Institut nicht auf fruchtbaren Boden fielen. Es waren nicht Desinteresse oder mangelnde Hilfsbereitschaft, vielmehr entsprach das Profil der Mitarbeiterinnen und Mitarbeiter - mit wenigen Ausnahmen - nicht den Anforderungen, die von jener Seite gestellt wurden. Dieses 1993 übernommene Dilemma hat Holger Dathe den größten Teil seiner Amtszeit begleitet.

Im Juli 1996 war diese initiale Periode beendet, es gab sehr brauchbare Empfehlungen des Wissenschaftsrates zur Zukunft des DEI. Am Berliner Museum für Naturkunde sollte ein gemeinsames Institut für systematische und ökologische Entomologie geschaffen werden, das den Namen „Deutsches Entomologisches Institut“ trägt. Für den Leiter des Deutschen Entomologischen Instituts wurde die Einrichtung einer C4-Stelle an der Humboldt-Universität empfohlen. In der Folge kam es jedoch durch Widerstände vor allem auf politischer Ebene nicht zur Umsetzung der Empfehlungen. Erst zum Jahresanfang 2000 erreichte das DEI eine institutionelle Förderung, als es schließlich in einer „Brandenburger Lösung“ dem Leibniz-Zentrum für Agrarlandschaftsforschung (ZALF) angegliedert wurde. Kernpunkte der Integration waren personelle Aufstockungen des DEI und ein Institutsneubau am Standort des ZALF in Müncheberg.

Mit dem Umzug des Deutschen Entomologischen Instituts nach Müncheberg, der schließlich 2004 erfolgen konnte, waren große Erwartungen verbunden. Schließlich gab es ein neues Haus, hervorragend eingerichtet, viele Wünsche zur Ausstattung konnten erfüllt werden. Die Vorbereitung dieser Phase ließ Holger Dathe nun zusätzlich zu den Fahrten nach Berlin noch zwischen Eberswalde und Müncheberg pendeln, bis diese Belastung durch den Umzug nach Waldsieversdorf, später nach Müncheberg, gemildert wurde. Endlose Verhandlungen, Sitzungen, viele Papiere, Evaluierungen ließen dem Chef kaum Zeit für eigene wissenschaftliche Arbeit. Oft fehlte sie oder - öfter noch - wurde sie ständig zerrissen, in kleine Segmente zerlegt, wo wir doch alle wissen, wie wichtig Kontinuität ist.

Die Absprachen wurden mit Ausnahme eines Institutsneubaus nicht eingelöst. Zum Zeitpunkt der Eingliederung hatte derjenige ZALF-Direktor, mit dem die Verhandlungen geführt worden waren, kurzfristig die Einrichtung verlassen. Weder konnten zusätzlich Ökologen eingestellt werden noch erhielt Holger Dathe die zugesagte Professur in Potsdam. Vielmehr wurde 
das Institut geschrumpft. Planstellen, deren Inhaber in Rente gingen bzw. nach außen berufen wurden, durften nicht wieder besetzt werden oder gingen sogar in den Bestand anderer Institute über. Bis heute ist z. B. das Lepidoptera-Kustodiat unbesetzt und das Institut damit weitgehend von der Entwicklung der Lepidopterologie abgeschnitten. Hinzu kamen Forderungen nach landschaftsökologischer Forschung oder wenigstens der Teilnahme an derartigen Projekten. Das ging natürlich nicht, denn die Mitarbeiterinnen und Mitarbeiter waren gar nicht für derartige Themen qualifiziert. Seriöse ökologische Forschung kann man nicht „nebenher mit“ machen. Strukturdiskussionen im ZALF gingen bis zur Eingliederung des DEI in andere ZALF-Institute oder sogar zu seiner Auflösung. Diese Situation führte zu ständigen Spannungen mit der Leitung des ZALF. Holger Dathe hat viel Zeit und Kraft verwendet, um die Realität einigermaßen erträglich zu halten, er stand häufig zwischen administrativen Zwängen und den entgegengesetzten Forderungen der Mitarbeiter, er musste vertrösten und beschwichtigen.

Es war abzusehen, dass hier der Wunsch nach grundlegender Veränderung aufkam, das DEI wieder in die Gemeinschaft der Leibniz-Forschungsmuseen zurückzuführen. Unter mehreren Möglichkeiten kristallisierte sich schließlich der Anschluss an den Senckenberg Forschungsverbund als besonders erstrebenswert heraus. Senckenberg hatte z. B. die Forschungsstation für Quartärpaläontologie in Weimar in seinen Bestand aufgenommen, sodass ein Modell vorlag. Außerdem liefen Verhandlungen mit den Dresdener Museen für Tierkunde und Mineralogie und dem Naturkundemuseum in Görlitz. Senckenberg begründete ein überregional tätiges, national organisiertes Forschungsmuseum mit weitem Profil. In diesen Prozess klinkte sich Holger Dathe mit dem Deutschen Entomologischen Institut ein und erreichte - auch hier wieder nicht ohne Kampf-, dass es am 1. Januar 2009 als Senckenberg Deutsches Entomologisches Institut (SDEI) Mitglied der Senckenberg Gesellschaft für Naturforschung wurde.

Damit waren die Grundprobleme gelöst, die allgemeine Freude groß. Bald aber begann der Vorgang des Zusammenwachsens, und das bedeutete für Holger Dathe eine neue enorme Herausforderung; schließlich waren seine Tage als Direktor, des Geburtsjahrgangs wegen, gezählt. Es lag ihm vor allem daran, für die Zukunft noch Weichen zu stellen, die dem Deutschen Entomologischen Institut unter den gewonnenen günstigen Voraussetzungen die bestmögliche Perspektive eröffnen. Das Institut sollte seiner historischen Weltgeltung für die Entomologie auch in den folgenden Jahrzehnten gerecht werden, manche Positionen waren auch zurück zu erobern.

Betrachtet man die Situation in den vier ausschließlich der Rezentzoologie verpflichteten Einrichtungen innerhalb des Senckenberg Forschungsverbundes, so ist es nicht unangemessen, eine Führungsrolle für die Entomologie in Müncheberg zu beanspruchen. Bibliothek, Sammlung, Mitarbeiterstab und Tradition sprechen eine unmissverständliche Sprache. Holger Dathe erarbeitete eine entsprechende Konzeption zur weiteren Entwicklung der Entomologie bei Senckenberg, die nach ausführlicher Diskussion und sachlicher Abstimmung gemeinsam zügig umgesetzt werden sollte. In der Praxis stellte sich das Vorhaben nicht so einfach dar, es wurde Zeit verloren, und alle Probleme werden schließlich an seinen Nachfolger übergehen. Bedauerlich ist vor allem, dass die offenen Stellen mit großer Verzögerung besetzt werden. Eine der größten und bedeutendsten Sammlungen der Welt an Coleoptera mit allem dazugehörigen Hinterland ist nach über 120 Jahren das erste Mal unbesetzt! Als Lothar Dieckmann dem Ruhestand nahe war, konnte die letzten drei Dienstjahre (1982-1985) sein designierter Nachfolger Lothar Zerche gleichzeitig im Deutschen Entomologischen Institut arbeiten. Das war eine weitschauende Entscheidung, die die Koleopterologie im Ganzen in hohem Maße befruchtet hat. Ähnlich dringend ist die Situation im Lepidopteren-Kustodiat, das seit 2005 unbesetzt ist. Es wäre Holger Dathe sehr zu wünschen gewesen, wenn das Deutsche Entomologische Institut zum Ende seiner Amtszeit 
eine größere Geschlossenheit und Ausgewogenheit aufweisen würde, dass er noch hätte gestalten können, was er mit so großem Einsatz für die Integration „seines“ Instituts in den Senckenberg Forschungsverbund vorbereitet hat. Natürlich mindert das Fehlen dieser Abrundung nicht die langjährig erfolgreiche Gesamtleistung seines Direktors.

Im bisher Gesagten konnte natürlich nicht alles angesprochen werden, was Holger Dathe für das Deutsche Entomologische Institut getan hat. Auf einige Aspekte soll aber noch hingewiesen werden.

Zum einen hat ihn der Gedanke sehr beschäftigt, dass die Restitution des Deutschen Entomologischen Instituts aus einer Projektgruppe in die nationale und internationale Forschergemeinschaft auf hoher Ebene betrieben werden muss. Entsprechend brachte er das DEI in zahlreiche Gremien und Gesellschaften immer wieder ein. So bemühte er sich intensiv, das Deutsche Entomologische Institut mit der universitären Lehre zu verbinden. Initiativen im Hinblick auf Potsdam haben nicht zum Erfolg geführt. Alle Hoffnungen richten sich seit einiger Zeit auf die Universität in Halle (Saale). Die hervorragende Zusammenarbeit mit Gerald Moritz hat bisher noch nicht zu einem abschließenden Ergebnis geführt, obwohl die Beteiligten mit Hartnäckigkeit und großem diplomatischem Geschick dieses lohnenswerte und in die Zukunft weisende Ziel verfolgt haben. Ständigen Kontakt zu den großen deutschen Forschungsmuseen hielt er über die Direktorenkonferenz Naturwissenschaftlicher Forschungsmuseen Deutschlands (DNFS). Er war hier ebenso Gründungsmitglied wie bei der Gesellschaft für Biologische Systematik (GfbS). Eine besonders enge Kooperation bestand darüber hinaus zur Zoologischen Staatssammlung München (hier war er Sprecher des Wissenschaftlichen Beirats), zum Museum Koenig in Bonn (Kooperationsvertrag) und - natürlich - zum Museum für Naturkunde Berlin.

Das zweite betrifft die „Beiträge zur Entomologie - Contributions to Entomology“, die unentbehrliche Institutszeitschrift. Hier gibt es mehrere Neuerungen, die auf das Wirken von Holger Dathe zurückgehen, z. B. die Einführung eines Peer-Review-Systems, besonders aber die Anbindung an die Deutsche Gesellschaft für allgemeine und angewandte Entomologie („Official Publication of the German Society of General and Applied Entomology"). Überhaupt ist seine Mitarbeit in dieser Gesellschaft hervorzuheben, als langjähriges Mitglied des Vorstandes, zuletzt auch als Stellvertretender Vorsitzender. Es geht auf seine Initiative zurück, dass die Geschäftsstelle der DGaaE dauerhaft am DEI eingerichtet wurde.

Drittens sei schließlich die Fortführung der Tradition der Förderung der Freizeitentomologie genannt, die im Deutschen Entomologischen Institut tief verwurzelt ist. In den „Beiträgen zur Insektenfauna der DDR“ hat sich dies ebenso niedergeschlagen wie in der „Entomofauna Germanica“. Beide Reihen wären ohne den fachlichen und logistischen Hintergrund des Instituts in der vorliegenden Form nicht möglich gewesen. Holger Dathe hat an dieser Stelle einerseits als Institutsdirektor, andererseits als stellvertretender Vorsitzender der Entomofaunistischen Gesellschaft e. V. fördernd gewirkt.

Holger Dathe hat es auch verstanden, wichtige Tagungen an das Deutsche Entomologische Institut zu holen. Ein besonderes Ereignis war sicher das XIX. Symposium Internationale Entomofaunisticum Europae Centralis (SIEEC) vom 5. bis 9. September 2005. Es war die 4. derartige Tagung, die in Deutschland stattfand. Holger Dathe war nicht nur Organisator dieses Symposiums, sondern er arbeitet sehr konstruktiv im Ständigen Internationalen Organisationskomitee dieser Symposien mit. Auch andere internationale Tagungen sind besonders hervorzuheben, so die Spezialistentreffen für Hymenoptera-Symphyta, Staphylinidae, Heteroptera, Diptera und etliche andere. Darüber hinaus war er auch bemüht, den Zwei-Jahres-Tagungen der Entomofaunistischen Gesellschaft im Deutschen Entomologischen Institut eine Heimstatt zu geben. Dreimal fanden sie unter seiner Gastgeberschaft statt. 
Seinen ständigen Bemühungen und hervorragenden Begründungen ist es schließlich zu danken, dass die Bestände der Bibliothek und die Insekten-Sammlungen seit 2009 als Geschütztes Kulturgut des Landes Brandenburg offiziell anerkannt wurden.

Zum äußeren Lebensgang von Holger Dathe finden sich Angaben in der Laudatio zum Festkolloquium anlässlich seines 60. Geburtstages (KLAusnitZer, 2005) sowie in einer Würdigung zum 65. Geburtstag (Klausnitzer, 2010). Im Anschluss an erstere Veröffentlichung wird hier das Publikationsverzeichnis weitergeführt, und es wird ein Verzeichnis der von ihm beschriebenen Taxa vorgelegt, ergänzt durch eine Liste der nach ihm benannten Arten.

Nun ist der Platz gekommen, von Herzen gute Wünsche für die Zukunft zu äußern. Mögen das Senckenberg Deutsche Entomologische Institut und das neue Arbeitszimmer so oft wie möglich von seiner Anwesenheit erfüllt sein und möge er - zwar nie ohne Sorgen um das Institut - aber doch mit mehr Ruhe seinen wissenschaftlichen Arbeiten nachgehen können, die großartigen Möglichkeiten in vollem Umfang nutzend, um deren Erhalt und Erweiterung er herausragende Verdienste hat! Die entomologische Welt dankt Prof. Dr. Holger H. Dathe in Hochachtung für seine umfassenden und uneigennützigen Leistungen um das Deutsche Entomologische Institut.

\section{Verzeichnis der Publikationen von Holger H. Dathe ab 2005}

130 Dathe, H. H. (Hrsg.) 2005: Lehrbuch der Speziellen Zoologie / begr. von Alfred Kaestner. Band I. Wirbellose Tiere, 5. Teil: Insecta. Korrigierter Nachdruck der 2. Aufl. - Heidelberg, Berlin: Spektrum Akademischer Verlag: XIV+ 961 S., 564 Abb. - ISBN 3-8274-0930-6.

131 Dathe, H. H. 2005: Deutschlands Hymenopterenfauna: Faunistik als Schlüssel zum Umweltverständnis. - Verh. XVIII. SIEEC Linz 2003. - Linzer biologische Beiträge, Linz 37 (1): 39-47.

132 Dathe, H. H. 2005: SIEEC XIX am Deutschen Entomologischen Institut in Müncheberg. Tagungsbericht. - Entomologische Nachrichten und Berichte, Dresden 49 (2): 159.

133 Dathe, H. H. 2005: Laudatio für Herrn Pfarrer P. Andreas Werner Ebmer anlässlich der Verleihung der Fabricius-Medaille 2005 der Deutschen Gesellschaft für allgemeine und angewandte Entomologie, 21. März 2005 in Dresden. - Wilhering, Stift und Gymnasium. 95. Jahresbericht 2004/2005: 13-16 [Vorabdruck aus: Mitteilungen der Deutschen Gesellschaft für allgemeine und angewandte Entomologie 15: 1-5 (2006)].

134 Dathe, H. H. 2005: Studien zur Systematik und Taxonomie der Gattung Hylaeus F. (4). Hylaeus sinensis sp. n. - Nachweis der montan-disjunkten Verbreitung einer Artengruppe um Hylaeus niger BRIDweLL (Apidae, Colletinae). - Beiträge zur Entomologie, Keltern 55 (2): 265-269.

135 Taeger, A. \& Dathe, H. H. 2005: Der Umzug des Deutschen Entomologischen Instituts nach Müncheberg. - Museum Aktuell, München. Nr. 121, Dez. 2005/Jan. 2006: 28-30.

136 Dathe, H. H. 2006: Tagungsberichte. SIEEC XIX vom 5. bis 9. September 2005 am Deutschen Entomologischen Institut in Müncheberg. - Entomologica Austriaca, Linz 13: 99-100.

137 Wagemann, H. (unter Mitarbeit von Dathe, H. H.) 2006: Deutsches Entomologisches Institut BerlinDahlem / Berlin-Friedrichshagen / Eberswalde / Müncheberg. - In: Wagemann, H. (Hrsg.), Von der Deutschen Akademie der Landwirtschaftswissenschaften zu Berlin zur Akademie der Landwirtschaftswissenschaften der DDR. Ein Beitrag zur Geschichte 1951-1991. Band 1/2, S. 74-93. - Berlin: Verlag am Park. - ISBN 3-89793-121-4.

138 Dathe, H. H. 2006: Glückwunsch zum 50. Jahrgang. - Entomologische Nachrichten und Berichte, Dresden 50 (1/2): 4

139 Dathe, H. H. (2006): Die historische Hymenopteren-Fauna des Plauenschen Grundes in Dresden nach Ludwig Heinrich Freiherrn von Block 1799 (Hym.). - Entomologische Nachrichten und Berichte, Dresden 50 (1/2): 35-44. 
140 Dathe, H. H. \& Groll, E. K. 2006: Editorial - In eigener Sache. - Beiträge zur Entomologie, Keltern 56 (1): $1-5$.

141 Dathe, H. H. 2006: Chronologische Tafel zur Geschichte des Deutschen Entomologischen Instituts 1886-2006. - Beiträge zur Entomologie, Keltern 56 (1): 7-24.

142 Dathe, H. H. 2006: Studien zur Systematik und Taxonomie der Gattung Hylaeus F. (5). Revision der Hylaeus-conformis-Gruppe (Apidae, Colletinae). - Beiträge zur Entomologie, Keltern 56 (1): 63-91.

143 Dathe, H. H. 2006: Laudatio für Herrn Pfarrer P. Andreas Werner Ebmer anlässlich der Verleihung der FABRICIUs-Medaille 2005 der Deutschen Gesellschaft für allgemeine und angewandte Entomologie am 21. März 2005 in Dresden. - Mitteilungen der Deutschen Gesellschaft für allgemeine und angewandte Entomologie 15: 1-5.

144 Dathe, H. H. \& Haszprunar, G. 2006: Preface / Geleitwort. - In: Blank, S. M.; Schmidt, S. \& TAeger, A. (eds), Recent Sawfly Research: Synthesis and Prospects: 7-8. - Keltern: Goecke \& Evers. - ISBN 3-931374-13-0.

145 Dathe, H. H. 2006: Willi Hennig. - In: Mason, F.; Stark, A. \& Tisato, M. (eds.), Calendar 2007. - CNBF Verona \& Studia dipterologica Halle a. S.

146 Dathe, H. H. 2007: Dr. Paul Westrich 60 Jahre. - Entomologische Nachrichten und Berichte, Dresden 51 (2): 151-154.

147 Dathe, H. H. 2007: Die ersten Jahre in Müncheberg. Vorwort: S. 5-7 - In: Jahresbericht 2005/2006 für das Deutsche Entomologische Institut, Müncheberg 2007.

148 Dathe, H. H. 2007: Vorwort. - In: Jacobs, H.-J., Die Grabwespen Deutschlands. Ampulicidae, Sphecidae, Crabronidae. - F. DAHL, die Tierwelt Deutschlands, 79. Teil, Hymenoptera III. Goecke und Evers, Keltern: 5-6. - ISBN 978-3-937783-33-8.

149 Dathe, H. H. 2007: Key to the Palearctic subgenera of Hylaeus. - In: Michener, C. D., The Bees of the World. 2. Auflage. Baltimore: Johns Hopkins University Press: 200-202.

150 Dathe, H. H. 2007: Zum Geleit. S. 7-10 - In: Kofferschläger, M., Unbedingt mausgrau. ScheunenVerlag 2007: 234 S., zahlr. Abb. - ISBN 978-3-938398-53-1.

151 Dathe, H. H. 2008: Zum zehnten Mal ein Insekt des Jahres: Insekten erweisen sich auch in dieser Aktion als erfolgreiche Tiergruppe. - Entomologische Nachrichten und Berichte, Dresden 52 (1): 1-3.

152 Dathe, H. H. 2008: Zum Jubiläum 150 Jahre Deutsche Entomologische Zeitschrift. - DGaaENachrichten 22 (1): 9-12.

153 Dathe, H. H. \& Groll, E. K. 2008: Editorial. - Beiträge zur Entomologie 58 (1): 1.

154 Petersen, G.; Groll, E. K. \& Dathe, H. H. 2008: Reinhard Gaedike zum 65. Geburtstag. Beiträge zur Entomologie 58 (1): 3-13.

155 Notton, D. G. \& Dathe, H. H. 2008: William Kirby's types of Hylaeus Fabricius (Hymenoptera, Colletidae) in the collection of the Natural History Museum, London. - Journal of Natural History 42 (27-28): 1861-1865. [ed. 18.08.2008]

156 Dathe, H. H. \& Groll, E. K. 2008: „Beiträge zur Entomologie“ ab 2008 Offizielle Publikation der DGaaE. - DGaaE-Nachrichten 22 (2): 114.

157 Dathe, H. H. 2008: Charles D. Michener 90 Jahre. - Beiträge zur Entomologie 58 (2): 476.

158 Dathe, H. H. 2008: Ehrenpromotion der Universität Greifswald für Bernhard Klausnitzer. Entomologische Nachrichten und Berichte, Dresden 52 (3-4): 225.

159 Dathe, H. H. 2009: Deutsches Entomologisches Institut. Forschungen zur Biodiversität bei Insekten seit 120 Jahren. - Natur und Museum, Frankfurt am Main 139 (1/2): 40-47.

160 Dathe, H. H. 2009: Die Gemeine Blutzikade. Insekt des Jahres 2009 Deutschland, Österreich, Schweiz. - Natur und Museum, Frankfurt am Main 139 (1/2): 48-49.

161 Dathe, H. H. 2009: Order Hymenoptera, superfamily Apoidea. Families Colletidae, Andrenidae, Halictidae, Melittidae, Megachilidae and Apidae. (With contributions by A. W. Ebmer; M. S. Engel; F. Gusenleitner; P. Hartmann; M. Kuhlmann;A. Müller; S. Risch;E. Scheuchl \&M. Schwarz). - In: van Harten. A., ed., Arthropod Fauna of the UAE. Vol. 2, pp. 335-432. - ISBN 978-9948-15-090-9. 
162 Dathe, H. H. 2009: Die Jahre in der Warteschleife. Vorwort: S. 4-8. - In: Jahresbericht 2007/2008 für das Deutsche Entomologische Institut, Müncheberg 2009.

163 Dathe, H. H. 2009: Ehrenpromotion der ERnst-Moritz-Arndt-Universität Greifswald für Bernhard KLAusnitZer. - Beiträge zur Entomologie, Keltern 59 (1): 1-2.

164 Engel, M. S. \& Dathe, H. H. 2009: In Memoriam: Donald Burton Baker (1922-2004). - Beiträge zur Entomologie 59 (1): 3-18.

165 Dathe, H. H.; Schubert, E. \& Taeger, A. 2009: Insektenforscher 3.0: Senckenbergs Entomologen geben Expertise online. - In: Leibniz Berlin Brandenburg. Forschung und Dienstleistung für die Wissenschaft, 32 mal Leibniz in der Region. Berlin: 60-61.

166 Dathe, H. H. \& Klausnitzer, B. (2009): Gerd Müller-Motzfeld 1941-2009 zu ehrendem Gedenken. - Beiträge zur Entomologie 59 (2): 263-264.

167 Dathe, H. H. 2009: Zur Festschrift für Bernhard Klausnitzer. - Entomologische Nachrichten und Berichte 53 (3/4): 145-149.

168 Dathe, H. H. 2009: Coleopterologie der Zukunft. Ehrenkolloquium für Bernhard Klausnitzer im Senckenberg DEI (Tagungsbericht). - Entomologische Nachrichten und Berichte 53 (3/4): 150.

169 Dathe, H. H. 2009: Anmerkungen zu Bau und Funktion sexualdimorpher Antennenstrukturen in der Gattung Hylaeus F. (Hymenoptera, Anthophila, Colletidae). - Entomologische Nachrichten und Berichte 53 (3/4): 151-156. - ed. 30.12.2009.

170 Klausnitzer, B. \& Dathe, H. H. 2009: Prof. Dr. Gerd Müller-Motzfeld (19. Juli 1941-24. Juli 2009) zu ehrendem Gedenken. - DGaaE-Nachrichten 23 (3): 148-151.

171 Chen, X.; Xu, H. \& Dathe, H. H. 2010: New subgenus of genus Hylaeus (Hymenoptera: Colletidae) with two new records from China. - Entomological Science 13 (1): 116-120.

172 Klausnitzer, B. \& Dathe, H. H. 2010: Dr. Lothar Zerche zum 65. Geburtstag. - Beiträge zur Entomologie 60 (1): 1-11.

173 Dathe, H. H. \& Seet, S. 2010: Insektenforscher 3.0: Senckenbergs Entomologen geben Expertise online: 60-61. - In: Leibniz Berlin Brandenburg. Forschung und Dienstleistung für die Wissenschaft. Hrsg. Leibniz-Gemeinschaft, Berlin.

174 Dathe, H. H. 2010: Studien zur Systematik und Taxonomie der Gattung Hylaeus F. (6). Arten asiatischer Hochgebirge und Anmerkungen zu weiteren asiatischen Arten (Hymenoptera, Anthophila, Colletidae). - Linzer biologische Beiträge 42 (1): 43-80.

175 Dathe, H. H. 2010: Die Insektenforschung - Entomologie von heute. - In: Gedanken zu einem Jahrzehnte-Programm für Natur- und Umweltschutz. Reden zur Festkonferenz anlässlich des 80. Geburtstages von Prof. Dr. Dr. h. c. Berndt Heydemann am 2.03.2010, Nieklitzer Ökologie- und Ökotechnologie-Stiftung (NICOL), Nieklitz: 27-29.

176 Dathe, H. H. 2010: Familie Apidae - Bienen. - In: Stresemann, E.; Hannemann, H.-J.; Klausnitzer, B. \& Senglaub, K. (Hrsg.). Klausnitzer, B. (Hrsg.), Exkursionsfauna von Deutschland 2. Wirbellose: Insekten. 11. Auflage. - Heidelberg: Spektrum.

\section{Neubeschreibungen: Arten der Gattung Hylaeus Fabricius}

nivaliformis Dathe, 1977, Hylaeus (Hylaeus). - Mitteilungen aus dem Zoologischen Museum in Berlin 53: 304-306. o 우, Italien: S-Tirol. Typus o ZMB

penalaris Dathe, 1979, Hylaeus (Dentigera). - Linzer biologische Beiträge 11 (1): 156-158. o o , Spanien. Typus ơ ZMB

acer Dathe, 1980, Hylaeus (Dentigera). - Entomologische Abhandlungen, Museum für Tierkunde Dresden 43 (5): 80-81, 86. ơ 9 , Iran. Typus ơ ZMB

crispulus DATHe, 1980, Hylaeus (Hylaeus). - Entomologische Abhandlungen, Museum für Tierkunde Dresden 43 (5): 84-85, 87. o $\diamond^{\star}$, Iran. Typus ơ ZMB 
ebmeri Dathe, 1980, Hylaeus (Hylaeus). - Entomologische Abhandlungen, Museum für Tierkunde Dresden 43 (5): 88, 90-91. ठ ${ }^{\star}$, Iran. Typus ơ ZMB

hyrcanius Dathe, 1980, Hylaeus (Prosopis), Entomologische Abhandlungen, Museum für

Tierkunde Dresden 43 (5): 79-80, 86. $0^{7}$ 9 , Iran. Typus ơ ZMB

implicatus Dathe, 1980, Hylaeus (Hylaeus). - Entomologische Abhandlungen, Museum für

Tierkunde Dresden 43 (5): 88, 91-92. o*, Iran. Typus ơ ZMB

iranicus Dathe, 1980, Hylaeus (Spatulariella). - Entomologische Abhandlungen, Museum für

Tierkunde Dresden 43 (5): 89, 93-94. o 우 , Iran. Typus ơ ZMB

irritans DATHE, 1980, Hylaeus (Spatulariella). - Entomologische Abhandlungen, Museum für

Tierkunde Dresden 43 (5): 94-95. 우, Iran. Typus 우 ZMB

jantaris Dathe, 1980, Hylaeus (Hylaeus). - Entomologische Abhandlungen, Museum für

Tierkunde Dresden 43 (5): 87-90. ơ 9 , Iran. Typus ơ ZMB

sariensis Dathe, 1980, Hylaeus (Lambdopsis). - Entomologische Abhandlungen, Museum für

Tierkunde Dresden 43 (5): 93. 오 , Iran. Typus ㅇ ZMB

koenigsmanni Dathe, 1981, Hylaeus (Hylaeus). - Linzer biologische Beiträge 13 (2): 146-149. ○

ㅇ, Griechenland. Typus ơ ZMB

altaicus Dathe, 1986, Hylaeus (Hylaeus). - Annales historico-naturales Musei Nationalis Hungarici

78: 277. đ', Mongolei. Typus ơ UNM Budapest

dorni Dathe, 1986, Hylaeus (Hylaeus). - Annales historico-naturales Musei Nationalis Hungarici

78: 294-295. o, Mongolei. Typus ơ MLU Halle

kaszabi Dathe, 1986, Hylaeus (Hylaeus). - Annales historico-naturales Musei Nationalis Hungarici

78: 289-291. $0^{\star}+$, Mongolei. Typus ơ UNM Budapest

montivagus Dathe, 1986, Hylaeus (Hylaeus). - Annales historico-naturales Musei Nationalis

Hungarici 78: 273-274. , Mongolei. Typus o MLU Halle

nimbatus Dathe, 1986, Hylaeus (Hylaeus). - Annales historico-naturales Musei Nationalis

Hungarici 78: 298. 우, Mongolei. Typus ㅇ MLU Halle

peregrinus Dathe, 1986, Hylaeus (Hylaeus). - Annales historico-naturales Musei Nationalis

Hungarici 78: 277-279. ㅇ, Mongolei. Typus o MLU Halle

pirus Dathe, 1986, Hylaeus (Hylaeus). - Annales historico-naturales Musei Nationalis Hungarici

78: 288-289. 우 , Mongolei. Typus ㅇ MLU Halle

stentoriscapus Dathe, 1986, Hylaeus (Hylaeus). - Annales historico-naturales Musei Nationalis

Hungarici 78: 271-273. ơ 오 , Mongolei. Typus ơ MLU Halle

stubbei Dathe, 1986, Hylaeus (Hylaeus). - Annales historico-naturales Musei Nationalis Hungarici

78: 296-297. $\sigma^{\star}$, Mongolei. Typus ơ MLU Halle

telmenicus Dathe, 1986, Hylaeus (Hylaeus). - Annales historico-naturales Musei Nationalis

Hungarici 78: 279-280. ㅇ, Mongolei. Typus o UNM Budapest

ulanus Dathe, 1986, Hylaeus (Hylaeus). - Annales historico-naturales Musei Nationalis Hungarici

78: 287-288. ㅇ, Mongolei. Typus ㅇ UNM Budapest

valinis DAthe, 1986, Hylaeus (Hylaeus). - Annales historico-naturales Musei Nationalis Hungarici

78: 296, 298. ㅇ, Mongolei. Typus o UNM Budapest

hohmanni Dathe, 1993, Hylaeus (Paraprosopis). - Veröff. Übersee-Museum Bremen (Nat.) 12:

749-751. o $0^{\top}$, Kanarische Inseln. Typus ơ Mus. ICN Sta. Cruz, Tenerife

aborigensis Dathe, 1994, Hylaeus (Hylaeus). - Beiträge zur Entomologie 44 (2): 442-445. o $~+$,

Russland: Sibirien. Typus ơ DEI 
donbakeri Dathe, 1995, Hylaeus (Paraprosopis). - Beiträge zur Entomologie 45 (1): 160-161. $o^{\star}$ i , Vereinigte Arabische Emirate. Typus o* DEI

fossifer DATHe, 1995, Hylaeus (Spatulariella). - Beiträge zur Entomologie 45 (1): 171-173. ơ $q$, Jordanien. Typus ơ DEI

hameri Dathe, 1995, Hylaeus (Hylaeus). - Beiträge zur Entomologie 45 (1): 168-170. ơ ․ Vereinigte Arabische Emirate. Typus o DEI

omanicus Dathe, 1995, Hylaeus (Paraprosopis). - Beiträge zur Entomologie 45 (1): 166-167. o, Vereinigte Arabische Emirate. Typus ơ DEI

convergens Dathe, 2000, Hylaeus (Prosopis). - Beiträge zur Entomologie 50 (1): 168-169. o ๆ, Spanien, Portugal. Typus ㅇ DEI

emir Dathe, 2000, Hylaeus (Paraprosopis). - Beiträge zur Entomologie 50 (1): 169-171. o, Vereinigte Arabische Emirate. Typus $0^{\star}$ DEI

hellenicus Dathe, 2000, Hylaeus (Hylaeus). - Beiträge zur Entomologie 50 (1): 154-158. ơ + ,

Griechenland. Typus o DEI

ibericus Dathe, 2000, Hylaeus (Hylaeus). - Beiträge zur Entomologie 50 (1): 160-162. ơ ๆ,

Spanien. Typus ơ DEI

pyrenaicus Dathe, 2000, Hylaeus (Hylaeus). - Beiträge zur Entomologie 50 (1): 162-164. ơ \%,

Spanien. Typus ơ DEI

tetris Dathe, 2000, Hylaeus (Koptogaster). - Beiträge zur Entomologie 50 (1): 171-172. o o ,

Türkei. Typus o DEI

sinensis Dathe, 2005, Hylaeus (Hylaeus). - Beiträge zur Entomologie 55 (2): 266-268. o, China.

Typus ơ Institute of Zoology, Academia Sinica, Beijing

maximilianus Dathe, 2006, Hylaeus (Dentigera). - Beiträge zur Entomologie 56 (1): 99-100. o,

Tunesien. Typus ơ DEI

sichuanensis Chen, Xu \& Dathe, 2010, Hylaeus (Laxoprosopis). - Entomological Science 13 (1):

117-119. $0^{\star}$ ㅇ , China. Typus ơ Institute of Zoology, Academia Sinica, Beijing

churtalis Dathe, 2010, Hylaeus (Hylaeus). - Linzer biologische Beiträge 42 (1): 45-46, 70. $\sigma^{\star}$,

Nepal. Typus ơ DEI

nepalensis Dathe, 2010, Hylaeus (Hylaeus). - Linzer biologische Beiträge 42 (1): 47-48, 71. ơ q,

Nepal. Typus o DEI

simikotalis Dathe, 2010, Hylaeus (Hylaeus). - Linzer biologische Beiträge 42 (1): 48-50, 71. o ๆ,

Nepal. Typus ơ DEI

persulcatus Dathe, 2010, Hylaeus (Hylaeus). - Linzer biologische Beiträge 42 (1): 50-51, 72. ㅇ, Nepal. Typus o DEI

deviatus Dathe, 2010, Hylaeus (Hylaeus). - Linzer biologische Beiträge 42 (1): 51-52, 72. o o ,

Nepal. Typus ơ DEI

kuhlmanni Dathe, 2010, Hylaeus (Hylaeus). - Linzer biologische Beiträge 42 (1): 53-55, 73. ơ,

Kasachstan. Typus ơ DEI

oehlkei Dathe, 2010, Hylaeus (Hylaeus). - Linzer biologische Beiträge 42 (1): 55-56, 74. o, Mongolei. Typus ơ DEI

creutzburgi Dathe, 2010, Hylaeus (Patagiata). - Linzer biologische Beiträge 42 (1): 56-57, 75. $\sigma^{\star}$ ㅇ , Nepal. Typus ơ DEI

karnaliensis Dathe, 2010, Hylaeus (Lambdopsis). - Linzer biologische Beiträge 42 (1): 58-59, 76.

o 오 , Nepal. Typus ơ DEI 
socheri Dathe, 2010, Hylaeus (Paraprosopis). - Linzer biologische Beiträge 42 (1): 61-63, 78. o Iran. Typus ơ DEI

taizzi Dathe, 2010, Hylaeus (Paraprosopis). - Linzer biologische Beiträge 42 (1): 63-65, 78. o ㅇ, Jemen. Typus ơ DEI

dubitzkyi Dathe, 2010, Hylaeus (Nesoprosopis). - Linzer biologische Beiträge 42 (1): 65-66, 79.

$\sigma^{*}+$, Taiwan. Typus $\sigma^{*}$ National University of Taichung, Republic of China

\section{Dedikationen}

Dolerus dathei Muche, 1973: 86-88. Kaukasus - Entomologische Nachrichten 17: 86-90.

Arachnotheutes dathei Wolf, 1981: 202. Mongolei - Mitteilungen aus dem Zoologischen Museum in Berlin 57: 193-211.

Elodes holgeri Klausnitzer, 2003: 199. Griechenland - Entomologische Nachrichten und Berichte 47: 199-202.

Scirtes dathei KLAuSnitzer, 2006: 160. Philippinen - Entomologica Basiliensia et Collectionis Frey 28: 155-174.

Lasioglossum (Evylaeus) dathei EвмеR, 2008: 5. VAE - Linzer biologische Beiträge 40: 551-580.

Sphecodes dathei ScHwARz, 2010: 483-486. VAE - Arthropod fauna of the UAE 3: 483-491.

Exochomoscirtes holgerdathei KLAusNitzer, 2010: 285. Indien - Beiträge zur Entomologie 60 (2): 281-293.

\section{Veröffentlichungen über Holger H. Dathe}

Klausnitzer, B. 2005a: Meigen-Medaille für Prof. Dr. Holger H. Dathe. - Entomologische Nachrichten und Berichte 49 (1): 14.

Klausnitzer, B. 2005b: Laudatio für Herrn Prof. Dr. Holger H. Dathe anlässlich seines 60. Geburtstages. - Beiträge zur Entomologie 55 (2): 253-264.

Klausnitzer, B. 2006: Laudatio für Herrn Prof. Dr. Holger H. Dathe anlässlich der Verleihung der Meigen-Medaille der DGaaE am 23. März 2005 in Dresden. - Mitteilungen der Deutschen Gesellschaft für allgemeine und angewandte Entomologie 15: 23-26.

Klausnitzer, B. 2010: Prof. Dr. Holger H. Dathe zum 65. Geburtstag. - Entomologische Nachrichten und Berichte 54 (2): 161-167.

\section{Anschrift des Verfassers:}

Prof. Dr. Dr. h. c. Bernhard Klausnitzer

Mitglied des Senckenberg Deutschen Entomologischen Instituts

Lannerstraße 5

01219 Dresden

E-Mail: klausnitzer.col@t-online.de 